\title{
PROOF OF THE FINITENESS OF MODULAR COVARIANTS*
}

\author{
BY \\ LEONARD EUGENE DICKSON
}

1. Let $f_{1}, \cdots, f_{l}$ be any system of forms (homogeneous polynomials) in the arbitrary variables $x_{1}, \cdots, x_{m}$ with undetermined integral coefficients taken modulo $p$, where $p$ is a prime. Let $c_{1}, c_{2}, \cdots$ denote the coefficients arranged in any order. Under the transformation

T:

$$
x_{i} \equiv \sum_{j=1}^{m} t_{i j} x_{j}^{\prime} \quad(\bmod p) \quad(i=1, \cdots, m),
$$

with integral coefficients, let $f_{i}$ become the form $f_{i}^{\prime}$, and let $c_{1}^{\prime}, c_{2}^{\prime}, \cdots$ denote the coefficients of $f_{1}^{\prime}, \cdots, f_{l}^{\prime}$ corresponding in position to $c_{1}, c_{2}, \cdots$, respectively. A rational integral function

$$
K\left(c_{1}, c_{2}, \cdots ; x_{1}, \cdots, x_{m}\right)
$$

with integral coefficients taken modulo $p$ is called a modular covariant of the forms $f_{1}, \cdots, f_{l}$ (under the group $G$ of all transformations $T$ ) if, for every transformation $T$,

$$
K\left(c_{1}^{\prime}, c_{2}^{\prime}, \cdots ; x_{1}^{\prime}, \cdots, x_{m}^{\prime}\right) \equiv\left|t_{i j}\right|^{\mu} K\left(c_{1}, c_{2}, \cdots ; x_{1}, \cdots, x_{m}\right) \quad(\bmod p)
$$

holds identically in $c_{1}, c_{2}, \cdots, x_{1}^{\prime}, \cdots, x_{m}^{\prime}$, after $x_{1}, \cdots, x_{m}$ are eliminated by means of the congruences $T$, and $c_{1}^{\prime}, c_{2}^{\prime}, \cdots$ are replaced by their expressions in terms of $c_{1}, c_{2}, \cdots \quad$ The exponent $\mu$ is called the index of $K$.

As an immediate generalization, we may take the coefficients $c_{i}$ of the forms $f_{1}, \cdots, f_{l}$, the coefficients $t_{i j}$ of the transformations $T$, and the coefficients of the covariant polynomials $K\left(c_{i} ; x_{j}\right)$ to be Galois imaginaries

$$
r_{0}+r_{1} \rho+r_{2} \rho^{2}+\cdots+r_{n-1} \rho^{n-1} \quad\left(r_{i}=0,1, \cdots, p-1\right),
$$

in which $\rho$ is a root of a fixed congruence of degree $n$, irreducible modulo $p$. The sum, difference, product or quotient (the divisor not being 0 ) of any two of these $p^{n}$ Galois imaginaries is one of these same imaginaries, so that they form a field of order $p^{n}$. This field is called the Galois field of order $p^{n}$ and designated $G F\left[p^{n}\right]$. For $n=1$, the field is that of the integral residues

* Presented to the Society (Chicago), March 22, 1913. 
modulo $p$; it is for this case that we gave above the explicit definition of modular covariants.

We do not consider in the present paper covariants of two or more sets of cogredient variables.

Any rational integral algebraic covariant with integral coefficients of a system of algebraic forms $f_{i}$ becomes a modular covariant of that system of forms when the coefficients of the $f_{i}$ are interpreted as arbitrary elements of any $G F\left[p^{n}\right]$. We obtain in this way only a relatively small proportion of the modular covariants. This fact is illustrated ( $\$ 7-13)$ by a complete set of fundamental covariants of the binary quadratic form in the $G F[3]$. Notwithstanding this greater prolixity of modular covariants, I obtain the

Theorem. The set of all modular covariants of any system of forms in $m$ variables is finite, in the sense that they are all rational integral functions, with coefficients in the basal field, of a finite number of the covariants of the set.

For $m=2$ the proof is very simple $(\S 2)$. For $m \geqq 3$ use is made of a lemma* on the finiteness of any set of monomial functions. The nature of the proof is best seen in the typical case $m=3(\S 3)$, when the functions and formulæ employed are quite simple and the lemma [the existence of relations (9)] is proved directly (without induction). The essential points in the extension to $m$ variables are given in $\$ 4$.

Finiteness of the modular covariants of binary forms.

2. By the theorem on the multiplication of two determinants of the second order, the function

$$
\left|\begin{array}{ll}
x^{p^{k n}} & y^{p^{k n}} \\
x & y
\end{array}\right|
$$

is an invariant of index unity under any linear homogeneous transformation on $x, y$, with coefficients in the $G F\left[p^{n}\right]$. Hence the group $G$ of all such transformations has the universal rational integral covariants $\dagger$

$$
L=x^{p^{n}} y-x y^{p^{n}}, \quad Q=\frac{x^{p^{2 n}} y-x y^{p^{2 n}}}{L}=x^{P}+\cdots \quad\left(P=p^{2 n}-p^{n}\right) ，
$$

$Q$ being an absolute covariant. We require also the fact that

$$
L=y \Pi(x-a y) \quad\left(a \text { ranging over the } G F\left[p^{n}\right]\right) .
$$

* In papers cited in \$ 4, I have recently employed this lemma to prove that there exist only a finite number of perfect or abundant numbers not multiples of smaller perfect or abundant numbers and having a given number of distinct odd prime factors and a given number of factors 2.

† Dickson, these Transactions, vol. 12 (1911), p. 1. It is there shown, in the spirit of finite projective geometry, that $L$ and $Q$ form a fundamental system of invariants of the group $G$. While this theorem is not presupposed in the present paper, it affords an explanation of the success attending the use here of $L$ and $Q$. 
A homogeneous covariant $K$, of order $\Omega$, of a system $\Sigma$ of binary forms shall be called regular or irregular according as its leader (the coefficients of $x^{\Omega}$ ) is not zero or zero. An irregular covariant has the factor $y$ and therefore, by (2), the factor $L$. The number of leaders $S$ of regular covariants is finite, since $S$ is a polynomial, with coefficients in the $G F\left[p^{n}\right]$, in the coefficients $c_{i}$ of the forms of the system $\Sigma$ and each $c_{i}$ has an exponent less than $p^{n}$.

All regular covariants with a given leader $S$ and orders $\Omega$ congruent modulo $P$ to a given integer $\omega$ shall be said to form the set $[S, \omega]$. Since $\omega$ may be restricted to $P$ or fewer values, and the number of leaders $S$ is finite, the number $f$ of sets is finite. Let

$$
k=S x^{\omega}+\cdots
$$

be a covariant of the set $[S, \omega]$ whose order $\omega$ is the least of the orders of covariants of this set. Then any new covariant of this set is of the form

$$
K=S x^{\omega+t P}+\cdots \quad(t \text { an integer } \geqq 0) .
$$

Hence $K-k Q^{t}$ is a covariant with the factor $y$ and hence an irregular covariant. Thus any regular covariant is of the form

$$
\phi\left(k_{1}, \cdots, k_{f}, Q\right)+I \text {, }
$$

where $\phi$ is a polynomial with integral coefficients in $Q$ and the covariants $k_{1}, \cdots, k_{f}$, one corresponding to each of the $f$ sets $[S, \omega]$, while $I$ is an irregular covariant. Now $I=L^{e} K^{\prime}$, where $K^{\prime}$ is a regular covariant. Expressing $K^{\prime}$ in the form (3) and treating $I^{\prime}=L^{e^{\prime}} K^{\prime \prime}$ similarly, we ultimately obtain the result that every covariant is a polynomial in $k_{1}, \cdots, k_{f}, Q, L$.* $^{*}$

\section{Finiteness of the modular covariants of ternary forms.}

3. The determinants, of which only the $i$ th column is written,

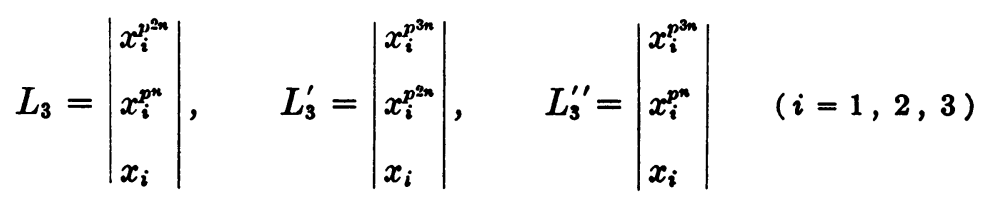

are invariants of index unity under any linear homogeneous transformation on $x_{1}, x_{2}, x_{3}$, with coefficients in the $G F\left[p^{n}\right]$. Hence the group $G$ of all such transformations has the universal rational integralt covariants

$$
L_{3}, \quad Q_{31}=L_{3}^{\prime} / L_{3}, \quad Q_{32}=L_{3}^{\prime \prime} / L_{3},
$$

* If the group is a subgroup $G^{\prime}$ of the total group $G$, the only modification in the proof is the replacement of $L$ by the product of the non-proportional linear functions into which $y$ is transformed by $G^{\prime}$.

† Dickson, these Trans a ctions, vol. 12 (1911), pp. 76, 77. The three form a fundamental system of invariants of the group $G$. 
$Q_{31}$ and $Q_{32}$ being absolute covariants. By considering the mirors of $x_{3}$ in the determinants (4), we find at once that, in the field,

(6) $Q_{32}=x_{1}^{P}+\cdots, Q_{31}=L^{E}+x_{3}(), P=p^{3 n}-p^{2 n}, E=p^{2 n}-p^{n}$, where

$$
L=x_{1}^{p n} x_{2}-x_{1} x_{2}^{p n}
$$

as in (1).

A homogeneous covariant $K$ of a system $\Sigma$ of ternary forms $f_{i}$ is called regular or irregular, according as it is not or is divisible by $x_{3}$. Thus an irregular covariant has the factor $L_{3}$ (see paper last cited).

Let $k$ be the sum of the terms of $K$ not involving $x_{3}$. Under a linear transformation on $x_{1}, x_{2}$ alone, let $k$ become $k_{1}$ and $f_{i}$ become $f_{i}^{\prime}$. By the covariancy of $K, k_{1}$ is identical apart from a constant factor with the function $k$ built for the coefficients and variables of the $f_{i}^{\prime}$. Hence if $k$ has the factor $x_{2}$ it has the factor $L$, given by (7). Thus

$$
k=L^{A}\left(S x_{1}^{B}+S_{1} x_{1}^{B-1} x_{2}+\cdots\right) \quad(S+0, A \geqq 0) .
$$

We shall call $S$ the leader of the regular covariant $K$.

Denote by $[S, a, b]$ the set of all the regular covariants

$$
K=S L^{A} x_{1}^{B}+\cdots, \quad A \equiv a(\bmod E), \quad B \equiv b(\bmod P),
$$

in which $S$ is a given leader $\neq 0$, while $a$ and $b$ are given integers. From this set we may select a finite number of covariants

$$
K_{i}=S L^{\Lambda_{i}} x_{1}^{B_{i}}+\cdots \quad(i=1, \cdots, t)
$$

such that, in any covariant (8) of the set,

$$
A \geqq A_{i}, \quad B \geqq B_{i} \quad \text { (for some value of } i \leqq t \text { ). }
$$

Indeed, we may take as $A_{1}$ the least $A$, and as $B_{1}$ the least $B$ occurring in the $L^{A_{1}} x_{1}^{B}$; as $B_{2}$ the least $B$, and as $A_{2}$ the least $A$ occurring in the $L^{A} x_{1}^{B_{2}}$. Hence, for a given covariant (8), relations (9) hold for $i=1$ or 2 , unless both $A<A_{2}$ and $B<B_{1}$. But the latter both hold only for a finite number of pairs of integers $A, B$, each $\geqq 0$, and these may be denoted by $A_{i}, B_{i}$ $(i=3, \cdots, t)$. Hence, by $(8)$, any covariant of the set $[S, a, b]$ has the form

$$
K=S L^{A_{\mathrm{i}}+l E} x_{1}^{B_{\mathrm{C}}+m P}+\cdots,
$$

where $l, m$ are integers $\geqq 0$ and $i$ is one of the integers $1, \cdots, t$. Hence, by (6), the terms free of $x_{8}$ in the covariant

$$
K^{\prime}=K-K_{i} Q_{31}^{l} Q_{32}^{m}
$$


have the factor $L^{\Lambda} x_{2}$. Thus $K^{\prime}$ is either irregular or is of the form

$$
S^{\prime} L^{A^{\prime}} x_{1}^{B^{\prime}}+\cdots, \quad S^{\prime} \neq 0, \quad A^{\prime}>A, \quad B^{\prime}<B .
$$

Let $K_{1}, \cdots, K_{\tau}$ serve for all the sets as did $K_{1}, \cdots, K_{t}$ for the particular set $[S, a, b]$. Thus any regular covariant has the form

$$
K=K_{i} Q_{31}^{l} Q_{32}^{m}+K^{\prime}
$$

where $K^{\prime}$ is either irregular or else is a regular covariant whose $B^{\prime}$ is less than the $B$ of $K$. In the latter case we have

$$
K^{\prime}=K_{j} Q_{31}^{\prime \prime} Q_{32}^{m^{\prime}}+K^{\prime \prime} \quad(j \leqq \tau),
$$

where $K^{\prime \prime}$ is either irregular or else is a regular covariant whose $B^{\prime \prime}$ is less than $B^{\prime}$. Hence, finally,

$$
K=f\left(K_{1}, \cdots, K_{\tau}, Q_{31}, Q_{32}\right)+I,
$$

where. $I$ is an irregular covariant, and $f$ is a rational integral function with integral coefficients. Now

$$
I=L_{3}^{c} K_{0},
$$

where $K_{0}$ is a regular covariant, possibly a constant. Expressing $K_{0}$ in the form (10), and repeating the process, we ultimately obtain $K$ expressed as a polynomial in $K_{1}, \cdots, K_{\tau}, Q_{31}, Q_{32}, L_{3}$.

\section{Finiteness of the modular covariants of m-ary forms.}

4. The determinants of order $m$

$$
\begin{array}{r}
L_{m}=\left|x_{i}^{p(m-1) n} \cdots x_{i}^{p n} x_{i}\right|, \quad L_{m}^{(s)}=\left|x_{i}^{p m n} \cdots x_{i}^{p(s+1) n} x_{i}^{p^{(s-1) n}} \cdots x_{i}\right| \\
(i=1, \cdots, m)
\end{array}
$$

are invariants of index unity under the group $G$ of all linear homogeneous transformations on $x_{1}, \cdots, x_{m}$ with coefficients in the $G F\left[p^{n}\right]$. The quotients $Q_{m}=L_{m}^{(s)} / L_{m}$ are integral functions in the field and are absolute universal covariants. By considering the minors of $x_{m}$, we find at once that

$L_{m}^{(1)} / L_{m}=L_{m-1}^{p_{m}^{2 m}-p^{n}}+x_{m}(), \quad L_{m}^{(s)} / L_{m}=\left(L_{m-1}^{(s-1)} / L_{m-1}\right)^{p^{n}}+x_{m}() \quad(s>1)$.

Using the second as a recursion formula, we get

$$
L_{m}^{(s)} / L_{m}=\left(L_{m-s+1}^{(1)} / L_{m-s+1}\right)^{p^{(8-1) n}}+f\left(x_{m-o+2}, \cdots, x_{m}\right),
$$

where every term of $f$ involves one of the variables displayed, but may involve also $x_{1}, \cdots, x_{m-1+1}$. Similarly for $f$ in (11) and (12). Hence, for any $s \leqq m-1$,

$$
Q_{m s}=L_{m-s}^{\pi_{0}}+f\left(x_{m-s+1}, \cdots, x_{m}\right), \quad \pi_{s} \equiv p^{(0+1) m}-p^{o n} .
$$


A more convenient notation proves to be

$$
q_{m s}=Q_{m m-s}=L_{s}^{P_{s}}+f\left(x_{s+1}, \cdots, x_{m}\right), \quad P_{\bullet}=\pi_{m-s} .
$$

Let $K$ be any regular covariant of a system $\Sigma$ of $m$-ary forms, $i$.e., one not divisible by $x_{m}$. Let $e_{m-1}$ be the exponent $\geqq 0$ of the highest power of $x_{m-1}$ which divides the sum $K_{m-1}$ of the terms of $K$ not involving $x_{m}$. Then

$$
K_{m-1}=L_{m-1}^{e_{m-1}}\left(K_{m-2}+x_{m-1} f\right),
$$

where $K_{m-2}$ is a function of $x_{1}, \cdots, x_{m-2}$ not identically zero. Let $e_{m-2}$ be the exponent $\geqq 0$ of the highest power of $x_{m-2}$ which divides $K_{m-2}$. Then

$$
K_{m-2}=L_{m-2}^{e_{m-2}}\left(K_{m-3}+x_{m-2} f^{\prime}\right) \quad\left(K_{m \rightarrow 0} \neq 0\right) .
$$

Proceeding similarly, and noting that $x_{1}=L_{1}$, we get

$$
K=S L_{1}^{e_{1}} L_{2}^{e_{2}} \cdots L_{m-1}^{e_{m-1}}+\cdots \quad(S \neq 0) .
$$

The coefficient $S$ is called the leader of the regular covariant $K$.

Denote by $\left[S, g_{1}, \cdots, g_{m-1}\right]$ the set of all the regular covariants (13) in which $S$ is a given leader and

$$
e_{1} \equiv g_{1}\left(\bmod P_{1}\right), \cdots, e_{m-1} \equiv g_{m-1}\left(\bmod P_{m-1}\right),
$$

where $g_{1}, \cdots, g_{m-1}$ are given integers. From this set we may select a finite number of covariants

$$
K_{i}=S L_{1}^{e_{i 1}} L_{2}^{e_{i \mathrm{~B}}} \cdots L_{m=1}^{\epsilon_{i m-1}}+\cdots \quad(i=1, \cdots, t) ，
$$

such that, in any covariant (13) of the set,

$$
\left.e_{1} \geqq e_{i 1}, \cdots, e_{m-1} \geqq e_{i m-1} \quad \text { ( for a certain } i \leqq t\right) .
$$

I have given elsewhere* a simple proof by induction of this lemma on a set of monomials forms in $m-1$ arbitrary variables $L_{1}, \cdots, L_{m-1}$. For $m=3$, a direct proof was given in $\S 3$. Thus any covariant of the set has the form

$$
K=S \prod_{j=1}^{m-1} L_{j}^{\varepsilon_{j}+r_{j} P_{j}}+\cdots \quad\left(r_{j} \text { integers } \geqq 0\right)
$$

The covariant, of the same order as $K$,

$$
K_{i} \prod_{j=1}^{m-1} q_{m j}^{r_{j}}
$$

has the same first term as $K$. Their difference either has the factor $x_{m}$, so that

$$
K=f\left(K_{1}, \cdots, K_{\tau}, q_{m 1}, \cdots, q_{m m-1}\right)+I \quad(I \text { irregular }),
$$

*American Journal of Mathematícs, October, 1913. 
for $\tau=t$, or is of the form

$$
\begin{array}{cc}
S^{\prime} L_{1}^{e_{1}^{\prime}} L_{2}^{e_{2}^{\prime}} \cdots L_{m-1}^{e_{m-1}^{\prime}}+\cdots & \left(S^{\prime} \neq 0\right), \\
e_{m-1}^{\prime}>e_{m-1} ; \quad \text { or } \quad e_{m-1}^{\prime}=e_{m-1}, \quad e_{m-2}^{\prime}>e_{m-2} ; \cdots ; & \cdots, \quad e_{3}^{\prime}=e_{3}, \quad e_{2}^{\prime}>e_{2},
\end{array}
$$

as shown by an inspection of the relations preceding (13).

Let $K_{1}, \cdots, K_{\tau}$ serve for all the sets as did $K_{1}, \cdots, K_{t}$ for the one set considered above. Thus any covariant is of the form (14) or

$$
K=f\left(K_{1}, \cdots, K_{\tau}, q_{m 1}, \cdots, q_{m m-1}\right)+K^{\prime},
$$

where $K^{\prime}$ is a regular covariant for which one of the alternatives (15) holds. Since $\Sigma d_{i} e_{i}$ is constant where $d_{i}$ is the total degree of $L_{i}$, repetitions of the process lead ultimately to a relation (14). Hence, as at the end of $\S 3$, every covariant is expressible as a polynomial in

$$
K_{1}, \cdots, K_{\tau}, q_{m 1}, \cdots, q_{m m-1}, L_{m} .
$$

Degrees and weights of the coefficients of a binary covariant.

5. Theorem. If $\mu$ is the index and $\omega$ the order of a homogeneous covariant $K$ of a binary form $f$ of order $q$ in the $G F\left[p^{n}\right]$, then $2 \mu+\omega$ is divisible by the greatest common divisor $d$ of. $q$ and $p^{n}-1$. The degrees of the various terms of the various coefficients of $K$ differ by multiples of $\left(p^{n}-1\right) / d$.

Let $\lambda$ be a primitive root in the field. Under the transformation

of determinant $\lambda^{2}$, let

$$
x=\lambda x^{\prime}, \quad y=\lambda y^{\prime}
$$

$$
\begin{aligned}
& f=a_{0} x^{q}+a_{1} x^{q-1} y+\cdots=a_{0}^{\prime} x^{q}+\cdots \quad .\left(a_{i}^{l}=\lambda^{q} a_{i}\right), \\
& K=S_{0} x^{\omega}+S_{1} x^{\omega-1} y+\cdots=\lambda^{\omega}\left(S_{0} x^{\prime \omega}+S_{1} x^{\prime \omega-1} y^{\prime}+\cdots\right) .
\end{aligned}
$$

Since $K$ is a covariant of index $\mu$,

$$
\begin{gathered}
\sum_{j=0}^{\infty} S_{j}\left(a_{i}^{\prime}\right) x^{\prime \omega-j} y^{\prime j}=\left(\lambda^{2}\right)^{\mu} K, \\
S_{j}\left(a_{i}^{\prime}\right)=\lambda^{2 \mu+\infty} S_{j}\left(a_{i}\right), \\
2 \mu+\omega \equiv q d_{t} \quad\left(\bmod p^{n}-1\right),
\end{gathered}
$$

where $d_{t}=e_{0}+\cdots+e_{q}$ is the degree of a term

$$
t=\tau a_{0}^{e_{0}} a_{1}^{e_{1}} \cdots a .
$$

of $S_{j}\left(a_{i}\right)$. The theorem follows from (16). 
Corollary. If $p>2$, there is no covariant of odd order $\omega$ of a binary form of even order $q$.

6. Theorem. The weight of any term of the leading coefficieni $S_{0}$ of a covariant $K$ differs from the index $\mu$ by a multiple of $p^{n}-1$.

Employing the transformation $x=x^{\prime}, y=\lambda y^{\prime}$, we get

$$
\begin{gathered}
a_{i}^{\prime}=\lambda^{i} a_{i}, \quad S_{j}\left(a_{i}^{\prime}\right)=\lambda^{\mu+j} S_{j}\left(a_{i}\right), \\
w_{j}=e_{1}+2 e_{2}+\cdots+q e_{q} \equiv \mu+j \quad\left(\bmod p^{n}-1\right) .
\end{gathered}
$$

Fundamental system of covariants of the binary quadratic form modulo 3 .

7. The linearly independent invariants (all absolute) of

$$
f=a x^{2}+2 b x y+c y^{2}
$$

under linear transformation modulo 3 are*

$$
\begin{aligned}
& 1, \Delta=b^{2}-a c, \quad I=\left(a^{2}-1\right)\left(b^{2}-1\right)\left(c^{2}-1\right), \\
& q=(a+c)\left(b^{2}+a c-1\right), \Delta^{2} .
\end{aligned}
$$

We require the seminvariants, viz., the invariants under

$$
x \equiv x^{\prime}+y^{\prime}, \quad y \equiv y^{\prime} \quad(\bmod 3) .
$$

The latter replaces $f$ by $f^{\prime}$, in which

$$
a^{\prime} \equiv a, \quad b^{\prime} \equiv a+b, \quad c^{\prime} \equiv a-b+c \quad(\bmod 3) .
$$

Hence the following functions are seminvariants:

$$
a, a^{2}, a \Delta, a \Delta^{2}, a^{2} \Delta, B=\left(a^{2}-1\right) b .
$$

Any seminvariant is a linear homogeneous function of the eleven linearly independent seminvariants (18) and (21). Indeed, $\dagger$ after subtracting constant multiples of these eleven, it remains only to consider a seminvariant

$$
S=\alpha_{1} b c^{2}+\alpha_{2} b c+\alpha_{3} b+\alpha_{4} b^{2} c^{2}+\alpha_{5} b^{2} c+\alpha_{6} b^{2}+\beta c^{2}+\gamma c,
$$

in which $\alpha_{1}, \alpha_{2}$ are linear functions of $a^{2}, a, 1$; and $\alpha_{3}, \cdots, \alpha_{6}$ are linear functions of $a, 1$; while the coefficients of these linear functions and $\beta, \gamma$ are constants (independent of $a, b, c)$. In the increment to $S$ under (20), the coefficient of $b c^{2}$ is $-a \alpha_{4}$, whence $\alpha_{4} \equiv 0$; then that of $b^{2} c$ is $\alpha_{1} \equiv 0$; then that of $b c$ is $\beta-a \alpha_{5}$, whence $\beta \equiv \alpha_{5} \equiv 0$; then that of $b^{2}$ is $-\alpha_{2} \equiv 0$; then that of $b$ is $-\gamma-a \alpha_{6}$, whence $\gamma \equiv \alpha_{6} \equiv 0$. Now $S=\alpha_{3} b$, whose increment is $\alpha_{3} a$, whence $\alpha_{3} \equiv 0$.

\footnotetext{
* These Tran s a c tio n s, vol. 8 (1907), p. 209. I now write $q$ for $Q$.

† A shorter, but less elementary, proof may be based upon the classes of forms $f$ under the group generated by.(19).
} 
Any polynomial in $\Delta, I, q, a, B$ may be expressed as a linear function of the eleven seminvariants (18), (21) by means of the relations

$$
\begin{gathered}
I^{2} \equiv-I, \quad q^{2} \equiv I-\Delta^{2}+1, \\
I \Delta \equiv I q \equiv I a \equiv I B \equiv q \Delta \equiv q B \equiv a B \equiv 0, \\
\Delta B \equiv B, \quad a^{2} \Delta^{2} \equiv \Delta^{2}+a^{2} \Delta-\Delta, \quad a q \equiv a^{2} \Delta^{2}-a^{2}, \quad B^{2} \equiv \Delta\left(1-a^{2}\right),
\end{gathered}
$$

and the evident relations $a^{3} \equiv a, \Delta^{3} \equiv \Delta$.

8. By the Corollary in $\S 5$, there is no covariant of odd order, We now determine the quadratic covariants

$$
C=S x^{2}+2 S_{1} x y+S_{2} y^{2} .
$$

Since $C$ - if is a covariant if $i$ is a constant multiple of one of the invariants (18), we may subtract from $S$ a constant multiple of $a, a \Delta, a \Delta^{2}$ or $a q$. By (22),

$$
a q=\Delta^{2}+a^{2} \Delta-\Delta-a^{2} .
$$

Hence, by $\$ 7$, we may assume that

$$
S=V+r a^{2}+s B
$$

( $V$ an invariant).

Under the transformation of determinant unity

$$
x \equiv y^{\prime}, \quad y \equiv-x^{\prime} \quad(\bmod 3),
$$

$f$ becomes $f^{\prime}$, in which $a^{\prime} \equiv \dot{c}, b^{\prime} \equiv-b, c^{\prime} \equiv a$. Hence

(25) $S_{1}(c,-b, a) \equiv-S_{1}(a, b, c), \quad S_{2} \equiv S(c,-b, a) \equiv V+r c^{2}+s B^{\prime}$,

$$
B^{\prime}=-\left(c^{2}-1\right) b \text {. }
$$

Under the transformation (19),

$$
C=S x^{\prime 2}+2\left(S+S_{1}\right) x^{\prime} y^{\prime}+\left(S+2 S_{1}+S_{2}\right){y^{\prime 2}}^{2}
$$

Since $C$ is to be a covariant, this must be identical with

$$
S^{\prime}{x^{\prime 2}}^{2}+2 S_{1}^{\prime} x^{\prime} y^{\prime}+S_{2}^{\prime}{y^{\prime 2}}^{2} \quad S_{i}^{\prime} \equiv S_{i}\left(a^{\prime}, b^{\prime}, c^{\prime}\right),
$$

where $a^{\prime}, \cdots$ are given by (20). Hence

$$
S^{\prime} \equiv S, \quad S_{1}^{\prime} \equiv S+S_{1}, \quad S_{2}^{\prime} \equiv S+2 S_{1}+S_{2} \quad(\bmod 3) .
$$

The first condition is satisfied since $S$ is a seminvariant. By the third condition and the invariance of $V$, we get

$$
S_{1} \equiv V+r(-\Delta-a b-b c)+s M, \quad M=\left(b^{2}+a c\right)(c-a) .
$$

By (251), we get

$$
-S_{1} \equiv V+r(-\Delta+a b+b c)-s M .
$$


Hence, by addition, $V=r \Delta$. Thus

$$
S_{1}=-r b(a+c)+s M, \quad M \equiv\left(b^{2}+a c\right)(c-a) .
$$

Since $\left(27_{2}\right)$ is seen to follow, $C$ is a covariant. For $r \equiv 0, s \equiv 1$, we get

$$
C_{1}=B x^{2}+2 M x y+B^{\prime} y^{2},
$$

a covariant of index unity. Indeed, for $x \equiv-x^{\prime}, y \equiv y^{\prime}$,

$C_{1}\left(a^{\prime}, b^{\prime}, c^{\prime}, x^{\prime}, y^{\prime}\right) \equiv-C_{1}\left(a, b, c, x^{\prime}, y^{\prime}\right), \quad a^{\prime} \equiv a, b^{\prime} \equiv-b, c^{\prime} \equiv c$.

For $r \equiv 1 s \equiv 0$, we get the absolute covariant

$$
C_{2}=\left(\Delta+a^{2}\right) x^{2}-2 b(a+c) x y+\left(\Delta+c^{2}\right) y^{2} .
$$

By use of (22), or by the table in $\S 13$, we find that

$$
\Delta C_{1} \equiv C_{1}, \quad q C_{1} \equiv 0, \quad \Delta C_{2} \equiv C_{2}+q f, \quad q C_{2} \equiv\left(\Delta^{2}-1\right) f
$$

9. We readily show that any covariant of order $6 t$ is of the form $P+L C$, where $C$ is a covariant of order $6 t-4, P$ is a polynomial in the covariants (including invariants) already found and $Q$, where, by (1),

$$
L=x^{3} y-x y^{3}, \quad Q=x^{6}+x^{4} y^{2}+x^{2} y^{4}+y^{6} .
$$

First, let $t$ be odd. Then the covariants

have as coefficients of $x^{6 t}$

$$
i f^{3 t}, i Q^{t}, C_{1}^{3 t}, C_{2}^{3 t}
$$

( $i$ an invariant )

$$
a i, i, B, \Delta+a^{2} \text {, }
$$

respectively. The linear combinations of the latter give all the seminvariants (18), (21). Next, let $t$ be even. Then

$$
f^{3 t}, \Delta f^{3 t}, i Q f^{3 t-3}, Q C_{1}^{3 t-3}, i_{1} Q^{t} \quad\left(i=1, \Delta, \Delta^{2} ; i_{1}=I, \Delta, \Delta^{2}, q 1\right)
$$

have as coefficients of $x^{6 t}$

$$
a^{2}, a^{2} \Delta, a i, B, i_{1}
$$

10. Lemma. If the order $\omega$ of a covariant $C$ of a binary quadratic form modulo 3 is not divisible by 3 , its leading coefficient $S$ is a linear homogeneous function of the seminvariants (18), (21), other than $1, I, q$.

Under the transformation (19),

$$
C=S x^{\omega}+S_{1} x^{\omega-1} y+\cdots \equiv S x^{\prime \omega}+\left(S_{1}+\omega S\right) x^{\prime \omega-1} y^{\prime}+\cdots
$$

For a covariant $C$, the final sum equals

$$
S x^{\prime \omega}+S_{1}^{\prime} x^{\prime \omega-1} y^{\prime}+\cdots, \quad S_{1}^{\prime} \equiv S_{1}\left(a^{\prime}, b^{\prime}, c^{\prime}\right),
$$


where $a^{\prime}, \cdots$ are given by (20). Hence

$$
S_{1}^{\prime}-S_{1} \equiv \omega S \quad(\bmod 3) \text {. }
$$

Set

Applying (20), we get

$$
S_{1}=k a^{2} b^{2} c^{2}+t \quad(t \text { of degree }<6) .
$$

$$
S_{1}^{\prime}=k a^{2}(a+b)^{2}(a-b+c)^{2}+t^{\prime} \equiv k a^{2}\left(a r+b^{2}+b c+b^{2} c^{2}\right)+t^{\prime},
$$

where $r$ is of degree 3 and $t^{\prime}$ of degree $<6$. Hence, by (33),

$$
\omega S \equiv k\left(a r+a^{2} b^{2}+a^{2} b c\right)+t^{\prime}-t(\bmod 3) .
$$

Since $\omega$ is prime to $3, S$ is of degree $<6$. Hence $S$ does not contain the term $a^{2} b^{2} c^{2}$, which occurs in $I$ but not in any other seminvariant (18), (21).

Next, if $S=1+\sigma$, where $\sigma$ is a function of $a, b, c$ without a constant term, $I C$ is a covariant $C^{\prime}$ with $S^{\prime}=I$.

Finally, let $S=q+\alpha_{1}+\alpha_{2} \Delta+\alpha_{3} \Delta^{2}+t B$, where $t$ is a constant and the $\alpha_{i}$ are functions of $a$. By (22),

$$
q S=I-\Delta^{2}+1+\alpha_{1} q,
$$

which has the term $a^{2} b^{2} c^{2}$ (from $I$ ).

11. Covariants $C$ of order $\omega=6 t+2$. For $t$ odd, the covariants

$$
f^{3 t+1}, Q^{t} f, C_{2}^{3 t+1}, f^{3 t} C_{2}, C_{1}^{3 t} C_{2}
$$

have as coefficients of $x^{\omega}$

$$
a^{2}, a, \Delta^{2}-a^{2} \Delta+a^{2}, a \Delta+a, B,
$$

respectively. Linear combinations of products of these by invariants give the seminvariants (21) and $\Delta^{2}, \Delta$. Hence, by the Lemma, $C=P+L C^{\prime}$, where $P$ is a polynomial in the known covariants. For $t$ even,

$$
f Q^{t}, f^{4} Q^{t-1}, C_{2} Q^{t}, C_{1} Q^{t}
$$

have $a, a^{2}, \Delta+a^{2}, B$ as coefficients of $x^{\omega}$.

12. Covariants $C$ of order $\omega=6 t+4$. The function

$$
f_{4}=a x^{4}+b x^{3} y+b x y^{3}+c y^{4} \text {, }
$$

which is congruent modulo 3 to the quadratic form $f$ for all integral values of $x, y$, is readily seen to be an absolute covariant (also when $a, b, c$ are arbitrary variables).

The coefficients of $x^{\infty}$ in the covariants

$$
f_{4} Q^{t}, f^{2} Q^{t}, C_{1} C_{2} Q^{t}, C_{1}^{2} Q^{t}
$$

are $a, a^{2}, B, \Delta-a^{2} \Delta$. Linear combinations of their products by invariants give all seminvariants not containing $1, I, q$. 
13. We have now completed the proof of the

Theorem. As a fundamental system of rational integral covariants of the binary quadratic form modulo 3 we may take

$$
\Delta, q, f, C_{1}, C_{2}, f_{4}, L, Q .
$$

No one of these eight concomitants is a rational integral function of the remaining seven. To prove this, consider their expressions for five special sets of values of $a, b, c$ (in fact, those giving the non-equivalent $f$ 's under the group of transformations of determinant unity):

\begin{tabular}{c|c|c|c|c|c|c} 
Case & $f$ & $\Delta$ & $q$ & $C_{1}$ & $C_{2}$ & $f_{4}$ \\
\hline 1 & 0 & 0 & 0 & 0 & 0 & 0 \\
2 & $x^{2}$ & 0 & -1 & 0 & $x^{2}$ & $x^{4}$ \\
3 & $-x^{2}$ & 0 & 1 & 0 & $x^{2}$ & $-x^{4}$ \\
4 & $x^{2}+y^{2}$ & -1 & 0 & 0 & 0 & $x^{4}+y^{4}$ \\
5 & $2 x y$ & 1 & 0 & $-x^{2}+y^{2}$ & $x^{2}+y^{2}$ & $x^{3} y+x y^{3}$
\end{tabular}

To show that $L$ and $Q$ are not functions of the remaining concomitants, we use case 1. For $f_{4}$, use case 4 . No linear relation holds between $f, C_{1}, C_{2}$ in which $C_{1}$ is present, since $C_{1}$ is of index 1 , while $f$ and $C_{2}$ are absolute covariants. Now $f \neq k C_{2}$ by case $4 ; C_{2} \neq k f$ by case 5 . Next, $q \neq F(\Delta)$ by 2 and $3 ; \Delta \neq F(q)$ by 4 and 5 .

We note the syzygies

$$
\begin{aligned}
f C_{1} & =2\left(\Delta^{2}+\Delta\right) L, & f C_{2} & =(1+\Delta) f_{4}, \\
C_{2}^{2}-C_{1}^{2} & =(\Delta+1)^{2} f^{2}, & C_{2}^{3}-f f_{4} & =\Delta Q .
\end{aligned}
$$

In the algebraic theory, $\Delta$ and $f$ form a fundamental system.

The Untversity of Chicago, January, 1913. 